\title{
Bone marrow aspirate: a viable source of stem cells for bone regeneration. A
}

\section{systematic review}

\author{
Aspirado de medula óssea: uma fonte viável de células-tronco para regeneração óssea. Uma revisão \\ sistemática
}

Aspirado de médula ósea: una fuente viable de células madre para la regeneración ósea. Una revisión sistemática

Received: 08/10/2021 | Reviewed: 08/18/2021 | Accept: 08/23/2021 |Published: 08/24/2021

Carolina dos Santos Santinoni

ORCID: https://orcid.org/0000-0001-5153-2419

University of Western Sao Paulo, Brazil

E-mail: carolsantinoni@msn.com

Yara Loyanne de Almeida Silva Levi

ORCID: https://orcid.org/0000-0003-1566-5673

University of Western Sao Paulo, Brazil

E-mail: yara-loyanne@ hotmail.com

João Paulo Pelágio Toneto

ORCID: https://orcid.org/0000-0002-8472-7009 University of Western Sao Paulo, Brazil

E-mail: doquitor@hotmail.com

João Augusto Cazuza

ORCID: https://orcid.org/0000-0003-2635-0730 University of Western Sao Paulo, Brazil

E-mail: gutocazuza@hotmail.com

Luciana Prado Maia

ORCID: https://orcid.org/0000-0001-5697-2587 University of Western Sao Paulo, Brazil

E-mail: lucianapmaia@gmail.com

Fellippo Ramos Verri

ORCID: https://orcid.org/0000-0001-5688-1669

University of Western Sao Paulo, Brazil

E-mail: fellippo.verri@unesp.br

\begin{abstract}
This systematic review evaluated the effectiveness of bone marrow aspirate (BMA) to enhance bone repair in humans. Comprehensive survey of ramdomized clinical trials published up to June 2021 and listed in PubMed/MEDLINE, EMBASE, and Cochrane Library databases following the Preferred Reporting Items for Systematic Reviews and Meta-Analyses (PRISMA) statement. Two reviewers independently searched eligible studies, made a final article selection, and extracted the data of the selected studies to evaluate it qualitatively. Overall, 13 studies were included in the review. Experimental models involved Posttraumatical aseptic nonunions of long bones of the upper limb, alveolar ridges following tooth extraction, atrophic mandibular fracture, benign bone lesions, bilateral tibial lengthening, fracture of intracapsular neck femur, maxillary horizontal ridge augmentation, non-traumatic femoral head necrosis, and sinus maxillary augmentation. The analyses included radiography, tomography, biopsies, and clinical evaluations. Ten studies reported enahanced bone formation (primary outcome) with combined use or not of BMA with other biomaterials and three studies found no benefit resulting from the use of BMA to treat bony defects. Secundary outcomes related to the healing process were also evaluated and positive, such as postoperative complications and pain visual analogue score. Within the limits of the present study, it can be concluded that BMA can improve the early stages of bone healing process.
\end{abstract}

Keywords: Bone marrow; Bone regeneration; Bone; Systematic review; Stem cells.

\section{Resumo}

Esta revisão sistemática avaliou a eficácia do aspirado de medula óssea (AMO) para melhorar o reparo ósseo em humanos. Pesquisa abrangente de ensaios clínicos ramificados publicados até junho de 2021 e listados nos bancos de dados PubMed / MEDLINE, EMBASE e Cochrane Library seguindo a declaração de itens de relatório preferidos para revisões sistemáticas e meta-análises (PRISMA). Dois revisores pesquisaram independentemente estudos elegíveis, fizeram uma seleção final do artigo e extraíram os dados dos estudos selecionados para avaliá-los qualitativamente. 
No geral, 13 estudos foram incluídos na revisão. Modelos experimentais envolveram não união asséptica póstraumática de ossos longos do membro superior, cristas alveolares após extração de dente, fratura mandibular atrófica, lesões ósseas benignas, alongamento tibial bilateral, fratura de fêmur do colo intracapsular, aumento da crista horizontal maxilar, necrose da cabeça femoral não traumática, e aumento do seio maxilar. As análises incluíram radiografia, tomografia, biópsias e avaliações clínicas. Dez estudos relataram formação óssea aprimorada (desfecho primário) com o uso combinado ou não de AMO com outros biomateriais e três estudos não encontraram nenhum benefício resultante do uso de AMO para tratar defeitos ósseos. Desfechos secundários relacionados ao processo de cicatrização também foram avaliados e positivos, como complicações pós-operatórias e escore visual analógico de dor. Dentro dos limites do presente estudo, pode-se concluir que a AMO pode melhorar as fases iniciais do processo de consolidação óssea.

Palavras-chave: Medula óssea; Regeneração óssea; Osso; Revisão sistemática; Células-tronco.

\section{Resumen}

Esta revisión sistemática evaluó la efectividad del aspirado de médula ósea (AMO) para mejorar la reparación ósea en humanos. Encuesta exhaustiva de ensayos clínicos aleatorios publicados hasta junio de 2021 y enumerados en las bases de datos de PubMed / MEDLINE, EMBASE y Cochrane Library siguiendo la declaración Preferred Reporting Items for Systematic Reviews and Meta-Analyzes (PRISMA). Dos revisores buscaron de forma independiente los estudios elegibles, seleccionaron el artículo final y extrajeron los datos de los estudios seleccionados para evaluarlos cualitativamente. En general, se incluyeron 13 estudios en la revisión. Modelos experimentales implicados Seudoartrosis aséptica postraumática de huesos largos del miembro superior, crestas alveolares después de extracción dentaria, fractura atrófica mandibular, lesiones óseas benignas, alargamiento tibial bilateral, fractura del fémur del cuello intracapsular, aumento del reborde horizontal maxilar, necrosis de la cabeza femoral no traumática, y aumento del seno maxilar. Los análisis incluyeron radiografías, tomografías, biopsias y evaluaciones clínicas. Diez estudios informaron una mejora en la formación de hueso (resultado primario) con el uso combinado o no de AMO con otros biomateriales y tres estudios no encontraron ningún beneficio como resultado del uso de AMO para tratar defectos óseos. Los resultados secundarios relacionados con el proceso de curación también se evaluaron y fueron positivos, como las complicaciones posoperatorias y la puntuación analógica visual del dolor. Dentro de los límites del presente estudio, se puede concluir que BMA puede mejorar las primeras etapas del proceso de curación ósea.

Palabras clave: Médula ósea; Regeneración ósea; Hueso; Revisión sistemática; Células madre.

\section{Introduction}

Surgery to reconstruct extensive bone defects is a huge challenge for professionals in orthopaedic field. Tissue engineering has been considered an emergent and alternative strategy for the treatment of these defects. In this context, a groing interest have been directed to bone marrow derived cells for regenerative therapies (Lana et al., 2021; Pelegrine et al., 2016; Kaigler et al., 2010).

Although autogenous bone graft remains the gold standard bone graft, it is associated with some disadvantages such high incidence of morbidity, prolonged operative time, blood loss, and length of hospitalization (Siegel et al., 2008). Osteoinduction growth factors and osteogenesis progenitor cells are some carachteristics of bone substitutes that are expected to achieve the potential healing of the autogenous graft (Siegel et al., 2008). A graft substitute that presents a scaffold and cells to promote osteogenesis from bone marrow aspirate (BMA) may reach the benefits of autogenous bone grafts without the undesirable points such as restrict availability (Siegel et al., 2008).

Evidence indicates that stem cells derived from bone marrow (BMSCs) have the potential to treat many disorders given their plasticity and ability to differentiate into various types of cells, including bone cells (Gupta et al., 2017; Badiavas and Falanga, 2013). Inflammatory response seems to be one important step in the bone repair process once it activates bone marrow cells and initiates the cascade of bone healing. BMSCs give rise to the cells that form mesenchymal tissues like bone and cartilage (Weel et al., 2015; Iser and Méndez-Ferrer, 2011; Pittenger et al., 1999).

Recent studies have evaluated the use of cultivated mesenchymal stem cells (MSC) derived from bone marrow to promote bone regeneration (Clough et al., 2015; Harada et al., 2014; He et al., 2014). The cultivation of MSC is a promising technique, but process involved in the in vitro expansion promotes loss of the original MSC phenotype resulting in significant implications for the development of regenerative therapies. The loss of the original phenotypic associated with in vitro 
cultivation has turned attention towards using unprocessed bone marrow or freshly isolated mononuclear cells (Bara et al., 2014). On a cellular level, using unprocessed BMSCs may preserve its capacities and abilities of multipotency, self-renew and homing. Using unprocessed cells can strength therapeutic potential and avoid treatment delay and extensive costs associated with cultivation steps (Bara et al., 2014). Therefore, studies have evaluated the use of BMA in bone healing, either alone or combined with other therapeutic modalities (Santinoni et al., 2021; Nagata et al., 2013; Bansal et al., 2009; Soltan et al., 2007), with promising results. Besides MSC, BMA contains a substantial amount of hematopoietic and endothelial stem cells (Smiler et al., 2008). However, it is also important to consider a decrease in the number and function of MSC in the bone marrow microenvironment when age related changes happen, and it can influence its ability of differentiate into osteoblasts and promote bone tissue formation (Duque, 2008). Furthermore, the number of MSC in bone marrow can also reduce in young individuals submitted to radiotherapy for cancer (Greenberger and Epperly, 2009) or ovariectomy in females performed before the natural age for menopause (Tewari et al., 2015). Thus, a reduction in endogenous MSCs due to advancing age or an underlying medical condition will also compromise the use of BMA as a rich source of these cells.

The present review was the first one to evaluate the clinical potential of BMA as a viable source of mesenchymal stem cells (MSC) to promote bone healing in human. Null hypothesis is there are no differences between bone defects treated with other biomaterials graft or untreated defects compared to bone marrow aspirate. Published scientific studies were reviewed to assess the information available on this topic to provide a more detailed understanding of the clinical effects of BMA on enhancing bone repair, and any other relevant circumstances regarding healing.

\section{Methodology}

This systematic review is based on the Preferred Reporting Items for Systematic Reviews and Meta-Analyses (PRISMA) checklist structure (Moher et al., 2010) and is in accordance with a model proposed in previously published reports (Oliveira et al., 2020; Santinoni et al., 2017; Lemos et al., 2016). Moreover, this study was registered on the international prospective register of systematic reviews (PROSPERO CRD42017069050). Two independent investigators (J.P.P. and J.A.C.) conducted an electronic search of PubMed/MEDLINE, EMBASE and Cochrane Library for articles published up to June 2021, using the following search terms: bone marrow aspirate AND bone healing OR bone marrow aspirate AND bone reapair OR bone marrow aspirate AND bone regeneration. They also conducted a search of the non-peer reviewed reports and currently unpublished registered trials. All differences in choices between the investigators were analyzed by a third investigator (F.R.V.), and consensus was reached through discussion.

Studies were independently selected and classified as included or excluded by the two researchers (J.P.P. and J.A.C.), based on the title and abstract of the articles. Eligible studies included randomized controlled trials (RCTs), studies that compared bone marrow aspirate and other treatments to promote bone formation, and studies published in English. Exclusion criteria were retrospective or prospective studies, in vitro or animal studies, computer simulations, case reports, studies that evaluated only one type of treatment without a comparison group, and published report reviews. A specific question was formulated based on the population, intervention, control, and outcome (PICO) criteria. The focused question was: "Bone marrow aspirate promotes bone regeneration?". Based on these criteria, the population was the participants who needed bone regeneration, the intervention was bone marrow aspirate, and the comparison was other biomaterials for bone graft. The primary outcome was bone formation, and secondary outcomes were other possible benefits observed and reported in the studies.

The Cochrane collaboration criteria for judging risk of bias were used to assess the quality of the studies included in the review (Figure 1). 
Two investigators (J.P.P. and J.A.C.) assessed the methodological quality of studies according to the Jadad scale, which ranges from 0 to 5, with studies that scored greater than or equal to 3 considered to be high quality (Jadad et al., 1996)

Figure 1. Assessing risk of bias in included studies by Cochrane risk of bias tools.

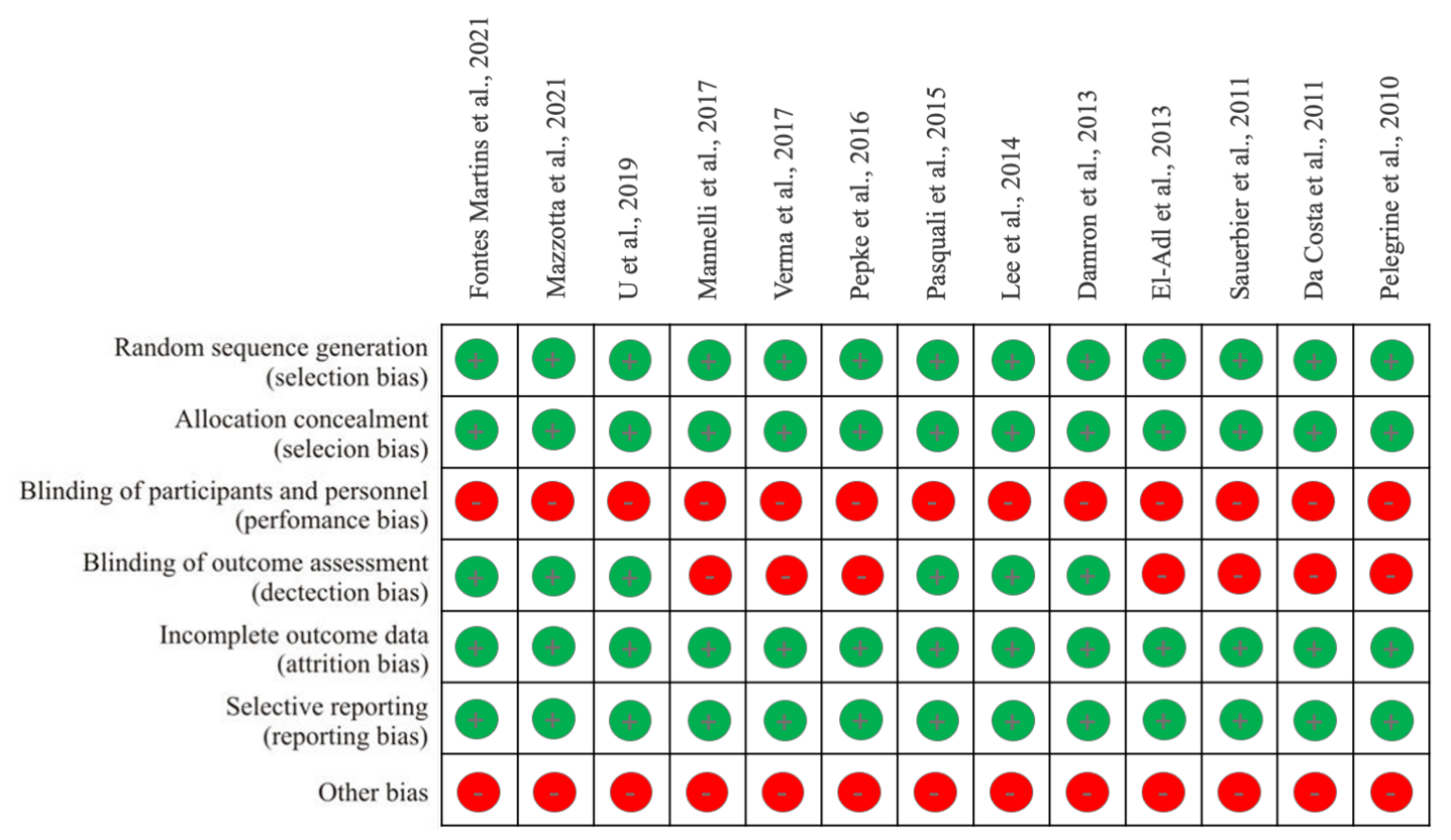

Source: Authors. 
Table 1. Quality assessment of the selected studies by JADAD scale.

\begin{tabular}{|c|c|c|c|c|c|c|c|c|c|c|c|c|c|}
\hline & & & & \multicolumn{10}{|c|}{ Studies } \\
\hline Quality criteria & 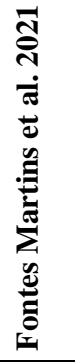 & 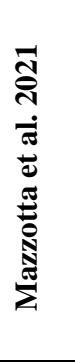 & 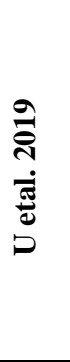 & 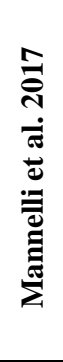 & 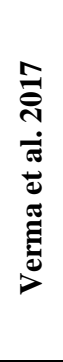 & 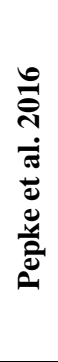 & 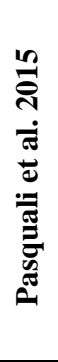 & 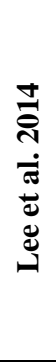 & 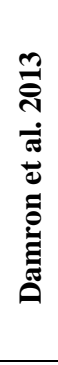 & 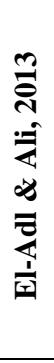 & 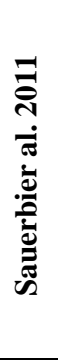 & 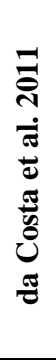 & 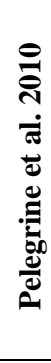 \\
\hline $\begin{array}{c}\text { 1. Was the study described as } \\
\text { random? }\end{array}$ & $\mathrm{Y}$ & $\mathrm{Y}$ & $\mathrm{Y}$ & $\mathrm{Y}$ & $\mathrm{Y}$ & $\mathrm{Y}$ & $\mathrm{Y}$ & $\mathrm{Y}$ & $\mathrm{Y}$ & $\mathrm{Y}$ & $\mathrm{Y}$ & $\mathrm{Y}$ & $\mathrm{Y}$ \\
\hline $\begin{array}{l}\text { 2. Was the randomization scheme } \\
\text { described and appropriate? }\end{array}$ & $\mathrm{Y}$ & $\mathrm{N}$ & $\mathrm{N}$ & $\mathrm{N}$ & $\mathrm{Y}$ & $\mathrm{Y}$ & $\mathrm{Y}$ & $\mathrm{Y}$ & $\mathrm{Y}$ & $\mathrm{Y}$ & $\mathrm{Y}$ & $\mathrm{N}$ & $\mathrm{N}$ \\
\hline $\begin{array}{c}\text { 3. Was the study described as } \\
\text { double blind? }\end{array}$ & $\mathrm{N}$ & $\mathrm{N}$ & $\mathrm{N}$ & $\mathrm{N}$ & $\mathrm{N}$ & $\mathrm{N}$ & $\mathrm{N}$ & $\mathrm{N}$ & $\mathrm{N}$ & $\mathrm{N}$ & $\mathrm{N}$ & $\mathrm{N}$ & $\mathrm{N}$ \\
\hline $\begin{array}{l}\text { 4. Was the method of double } \\
\text { blinding appropriate? }\end{array}$ & $\mathrm{N}$ & $\mathrm{N}$ & $\mathrm{N}$ & $\mathrm{N}$ & $\mathrm{N}$ & $\mathrm{N}$ & $\mathrm{N}$ & $\mathrm{N}$ & $\mathrm{N}$ & $\mathrm{N}$ & $\mathrm{N}$ & $\mathrm{N}$ & $\mathrm{N}$ \\
\hline $\begin{array}{l}\text { 5. Was there a description of } \\
\text { dropouts and withdrawals? }\end{array}$ & $\mathrm{Y}$ & Y & Y & $\mathrm{Y}$ & $\mathrm{Y}$ & $\mathrm{N}$ & $\mathrm{N}$ & $\mathrm{Y}$ & $\mathrm{N}$ & $\mathrm{N}$ & $\mathrm{N}$ & $\mathrm{N}$ & $\mathrm{N}$ \\
\hline Jadad score & 3 & 3 & 2 & 2 & 3 & 2 & 2 & 3 & 2 & 2 & 2 & 1 & 1 \\
\hline Quality of study & in & ton & $\stackrel{3}{9}$ & 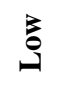 & 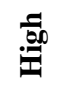 & $\stackrel{3}{9}$ & $\stackrel{3}{\varrho}$ & 包 & $\stackrel{3}{9}$ & $\stackrel{3}{9}$ & $\frac{1}{60}$ & $\stackrel{3}{9}$ & $\stackrel{3}{9}$ \\
\hline
\end{tabular}

Abrevitations: Y, Yes; N, No. Source: Authors.

\section{Results}

\subsection{Literature search}

The database search retrieved 87 references. After applying the inclusion/exclusion criteria to the titles and abstracts of the selected comparative studies, 14 studies remained. Reading these study texts resulted in exclusion of 1 more study because it was not controlled (Jäger et al., 2011). The manual search for articles did not identify more studies. Details of the search strategy are presented in Figure 2. Overall, 13 studies (Fontes Martins et al., 2021; Mazzotta et al., 2021; U et al., 2019; Manelli et al., 2017; Verma et al., 2017; Pepke et al., 2016; Pasquali et al., 2015; Lee et al., 2014; Damron et al., 2013; Ed-Adl et al., 2013; Sauerbier et al., 2011; Da Costa et al., 2011) were selected for the analysis (Tables 1 and 2).

The kappa inter-investigator agreement for articles that were selected from databases (kappa value $=0.83$ ) showed an almost perfect agreement (Landis \& Koch, 1977). 
Figure 2. Literature search diagram flow.

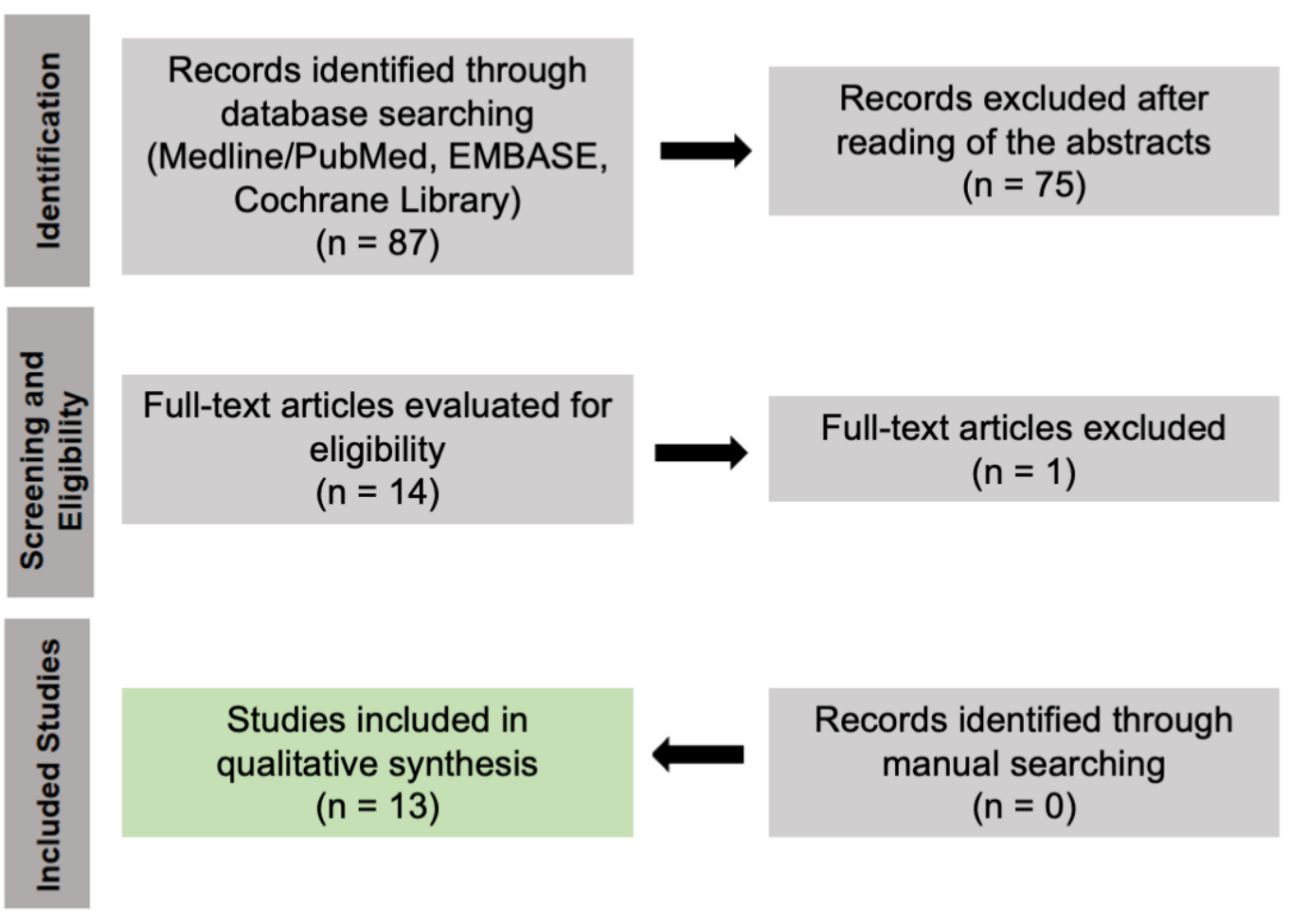

Source: Authors. 
Table 2. Qualitative characteristics of the included studies related to patients, treatments and results.

\begin{tabular}{|c|c|c|c|c|c|c|c|c|c|c|}
\hline Author & $\mathbf{n}$ & $\begin{array}{c}\text { Age } \\
\text { Mean } \\
\text { (years) }\end{array}$ & Systemic condition & $\begin{array}{l}\text { Experimental } \\
\text { model }\end{array}$ & $\begin{array}{c}\text { BMA } \\
\text { processing }\end{array}$ & $\begin{array}{l}\text { Combination } \\
\text { with another } \\
\text { biomaterials }\end{array}$ & $\begin{array}{c}\text { Result } \\
\text { analysis }\end{array}$ & $\begin{array}{l}\text { Parameter } \\
\text { evaluated }\end{array}$ & $\begin{array}{c}\text { Greater } \\
\text { Follow up } \\
\text { (months) }\end{array}$ & Results \\
\hline $\begin{array}{c}\text { Fontes } \\
\text { Martins et al. } \\
2021\end{array}$ & 15 & 47.13 & General good health & $\begin{array}{l}\text { Alveolar } \\
\text { ridges } \\
\text { following } \\
\text { tooth } \\
\text { extraction }\end{array}$ & $\begin{array}{l}400 \mathrm{~g}, \\
12 \mathrm{~min}\end{array}$ & $\begin{array}{l}\text { Platelet-rich } \\
\quad \text { fibrin }\end{array}$ & $\begin{array}{l}\text { Histological, } \\
\text { histometric, } \\
\text { and immuno- } \\
\text { histochemical } \\
\text { analyses }\end{array}$ & $\begin{array}{l}\text { Mineralized } \\
\text { tissue area, } \\
\text { osteocalcin } \\
\text { and Runx2 }\end{array}$ & 6 & $\begin{array}{l}\text { Significantly higher } \\
\text { percentage of } \\
\text { mineralized tissue and } \\
\text { osteocalcin expression }\end{array}$ \\
\hline $\begin{array}{l}\text { Mazzotta et } \\
\text { al. } 2021\end{array}$ & 68 & 44.15 & Not reported & $\begin{array}{l}\text { Posttraumatica } \\
1 \text { aseptic } \\
\text { nonunions of } \\
\text { long bones of } \\
\text { the upper limb }\end{array}$ & $\begin{array}{l}3200 \mathrm{rpm} \\
10 \mathrm{~min}\end{array}$ & $\begin{array}{l}\text { Lyophilized } \\
\text { bone chips and } \\
\text { platelet-rich } \\
\text { fibrin }\end{array}$ & $\begin{array}{l}\text { Clinical and } \\
\text { radiographic } \\
\text { analyses }\end{array}$ & $\begin{array}{l}\text { First signs of } \\
\text { healing and } \\
\text { percentages of } \\
\text { radiological } \\
\text { healing }\end{array}$ & 24 & $\begin{array}{c}\text { Accelerate the healing } \\
\text { time }\end{array}$ \\
\hline U et al. 2019 & 30 & 35 & $\begin{array}{c}\text { Excluded patients } \\
\text { having any debilitating } \\
\text { disease, blood disorders, } \\
\text { allergies, neoplasia, or } \\
\text { were smokers }\end{array}$ & $\begin{array}{c}\text { Cystic } \\
\text { Maxillofacial } \\
\text { Bony Defects }\end{array}$ & None & $\begin{array}{l}\text { Hydroxyapatite } \\
\text { collagen } \\
\text { scaffold }\end{array}$ & $\begin{array}{l}\text { Clinical and } \\
\text { radiologic } \\
\text { assessment, } \\
\text { postoperative } \\
\text { pain and } \\
\text { swelling }\end{array}$ & $\begin{array}{c}\text { Pain using } \\
\text { visual analog } \\
\text { scale, swelling } \\
\text { using Laskin } \\
\text { method, tooth } \\
\text { mobility, } \\
\text { pus discharge, } \\
\text { wound gap, } \\
\text { extrusion of } \\
\text { graft, nerve } \\
\text { injury and } \\
\text { bone defect } \\
\text { volume. }\end{array}$ & 6 & $\begin{array}{l}\text { Early bone } \\
\text { regeneration, and faster } \\
\text { wound healing. }\end{array}$ \\
\hline
\end{tabular}


Research, Society and Development, v. 10, n. 11, e94101119265, 2021

(CC BY 4.0) | ISSN 2525-3409 | DOI: http://dx.doi.org/10.33448/rsd-v10i11.19265

\begin{tabular}{|c|c|c|c|c|c|c|c|c|c|c|}
\hline $\begin{array}{l}\text { Mannelli et al. } \\
2017\end{array}$ & 35 & 76.8 & $\begin{array}{l}\text { Excluded patients } \\
\text { presenting pathological } \\
\text { fractures, patients who } \\
\text { did not give their own } \\
\text { consent to be enrolled in } \\
\text { this study protocol, } \\
\text { patients with a previous } \\
\text { medical history of } \\
\text { mandible fractures, } \\
\text { patients who had } \\
\text { undergone head and } \\
\text { neck radiotherapy } \\
\text { treatment, and patients } \\
\text { with insufficient } \\
\text { preoperative or } \\
\text { postoperative data }\end{array}$ & $\begin{array}{l}\text { Atrophic } \\
\text { mandibular } \\
\text { fracture }\end{array}$ & $\begin{array}{l}1600 \mathrm{~g} \\
8 \mathrm{~min}\end{array}$ & $\begin{array}{l}\text { Platelet rich } \\
\text { plasm }\end{array}$ & $\begin{array}{c}\text { X Ray, } \\
\text { tomograph, } \\
\text { photographic } \\
\text { records and } \\
\text { pain visual } \\
\text { analogue } \\
\text { score }\end{array}$ & $\begin{array}{l}\text { Quality of } \\
\text { surgical } \\
\text { reduction, } \\
\text { bone } \\
\text { consolidation } \\
\text { and } \\
\text { postoperative } \\
\text { complications }\end{array}$ & 12 & $\begin{array}{l}\text { Bone marrow aspirate } \\
\text { cells is a safe and useful } \\
\text { procedure which has a } \\
\text { lower complication rate } \\
\text { when compared to } \\
\text { standard technique }\end{array}$ \\
\hline
\end{tabular}


Research, Society and Development, v. 10, n. 11, e94101119265, 2021

(CC BY 4.0) | ISSN 2525-3409 | DOI: http://dx.doi.org/10.33448/rsd-v10i11.19265

\begin{tabular}{|c|c|c|c|c|c|c|c|c|c|c|}
\hline $\begin{array}{c}\text { Pepke et al. } \\
2016\end{array}$ & 24 & 44.4 & $\begin{array}{l}\text { Included patients } \\
\text { submitted to } \\
\text { chemotherapy or } \\
\text { immunosuppressive } \\
\text { therapy }\end{array}$ & $\begin{array}{c}\text { Non-traumatic } \\
\text { femoral head } \\
\text { necrosis }\end{array}$ & $\begin{array}{c}\text { Sepax } \\
\text { centrifugati } \\
\text { on device } \\
\text { protocol }\end{array}$ & None & $\begin{array}{l}\text { X Ray and } \\
\text { pain visual } \\
\text { analogue } \\
\text { scale }\end{array}$ & $\begin{array}{l}\text { Head survival } \\
\text { rate and } \\
\text { volume of } \\
\text { non-traumatic } \\
\text { femoral head } \\
\text { necrosis }\end{array}$ & 24 & $\begin{array}{l}\text { It was not observed any } \\
\text { benefit from the } \\
\text { injection of bone } \\
\text { marrow concentrate } \\
\text { with regard to bone } \\
\text { regeneration and } \\
\text { clinical outcome in the } \\
\text { short term }\end{array}$ \\
\hline $\begin{array}{l}\text { Pasquali et al. } \\
2015\end{array}$ & 8 & 55 & $\begin{array}{l}\text { Excluded patients if they } \\
\text { had a history of } \\
\text { neoplastic disease } \\
\text { treated with radiotherapy } \\
\text { or chemotherapy, if they } \\
\text { were pregnant or } \\
\text { breastfeeding, if they } \\
\text { were receiving treatment } \\
\text { or were a ected by an } \\
\text { illness that could have } \\
\text { an e ect on bone } \\
\text { homeostasis, allergy to } \\
\text { any of the materials } \\
\text { used, and sinus } \\
\text { pathologies, or if they } \\
\text { were smokers }\end{array}$ & $\begin{array}{c}\text { Sinus } \\
\text { maxillary } \\
\text { augmentation }\end{array}$ & $14 \mathrm{~min}$ & $\begin{array}{l}\text { Combined or } \\
\text { not with } \\
\text { xenogeneic } \\
\text { bone from } \\
\text { bovine } \\
\text { hydroxyapatite }\end{array}$ & Biopses & $\begin{array}{l}\text { New bone } \\
\text { formation } \\
\text { (Histomorpho } \\
\text { metrical } \\
\text { analysis) }\end{array}$ & 6 & $\begin{array}{c}\text { Bone marrow } \\
\text { concentrate increased } \\
\text { bone formation in sinus } \\
\text { lifting procedures }\end{array}$ \\
\hline
\end{tabular}


Research, Society and Development, v. 10, n. 11, e94101119265, 2021

(CC BY 4.0) | ISSN 2525-3409 | DOI: http://dx.doi.org/10.33448/rsd-v10i11.19265

\begin{tabular}{|c|c|c|c|c|c|c|c|c|c|c|}
\hline $\begin{array}{l}\text { Lee et al. } \\
2014\end{array}$ & 20 & 21.5 & $\begin{array}{l}\text { No history of medical } \\
\text { illness, fracture, soft } \\
\text { tissue compromise, bony } \\
\text { deformities, or infections } \\
\text { of the lower extremity }\end{array}$ & $\begin{array}{l}\text { Bilateral tibial } \\
\text { lengthening }\end{array}$ & $\begin{array}{l}3200 \mathrm{rpm}, \\
15 \mathrm{~min}\end{array}$ & $\begin{array}{c}\text { BMA } \\
\text { concentrated } \\
\text { combined with } \\
\text { PRP }\end{array}$ & X Ray & $\begin{array}{l}\text { Latent period } \\
\text { (days); } \\
\text { Distraction } \\
\text { rate (mm/day); } \\
\text { Final length } \\
\text { gain (mm); } \\
\text { External } \\
\text { fixator index } \\
\text { (months/cm); } \\
\text { Intramedullary } \\
\text { nail diameter } \\
\quad \text { (cm); } \\
\text { Intramedullary } \\
\text { nail length } \\
\text { (cm); } \\
\text { Mean cortical } \\
\text { healing index } \\
\text { (months/cm); } \\
\text { Anterior } \\
\text { cortex; } \\
\text { Posterior } \\
\text { cortex; Medial } \\
\text { cortex; Lateral } \\
\text { cortex }\end{array}$ & 28 & $\begin{array}{l}\text { Improved bone healing } \\
\text { in distraction } \\
\text { osteogenesis of the } \\
\text { tibia, although the } \\
\text { effect size was small }\end{array}$ \\
\hline
\end{tabular}


Research, Society and Development, v. 10, n. 11, e94101119265, 2021

(CC BY 4.0) | ISSN 2525-3409 | DOI: http://dx.doi.org/10.33448/rsd-v10i11.19265

\begin{tabular}{|c|c|c|c|c|c|c|c|c|c|c|}
\hline $\begin{array}{c}\text { Damron et al. } \\
2013\end{array}$ & 55 & 23.5 & $\begin{array}{l}\text { No patients with a } \\
\text { known posttraumatic } \\
\text { defect were included. } \\
\text { Patients with active } \\
\text { infection, bone marrow } \\
\text { disorders, or other } \\
\text { contraindications to the } \\
\text { use of supplemental } \\
\text { bone marrow aspiration } \\
\text { as well as those who } \\
\text { preferred autologous or } \\
\text { allogeneic graft material } \\
\text { alone or declined follow- } \\
\text { up were excluded as } \\
\text { well }\end{array}$ & $\begin{array}{l}\text { Benign bone } \\
\text { lesion }\end{array}$ & None & $\begin{array}{l}\text { Ultraporous B- } \\
\text { tricaicium } \\
\text { phosphate }\end{array}$ & $\begin{array}{l}\mathrm{X} \text { Ray and } \\
\text { tomography }\end{array}$ & $\begin{array}{c}\text { Resorption of } \\
\text { Graft } \\
\text { Material, } \\
\text { Presence of } \\
\text { Rim of } \\
\text { Radiolucency } \\
\text { Surrounding } \\
\text { Grafted } \\
\text { Defect, } \\
\text { Trabeculation } \\
\text { Through the } \\
\text { Defect, } \\
\text { Persistence of } \\
\text { Graft Material } \\
\text { Through } \\
\text { Lesion, Size } \\
\text { defect }\end{array}$ & 19 & $\begin{array}{c}\text { Significant } \\
\text { improvements in } \\
\text { radiographic parameters } \\
\text { were observed in both } \\
\text { TCP groups over two } \\
\text { years of follow-up, but } \\
\text { the addition of BM was } \\
\text { not found to provide } \\
\text { any significant benefit }\end{array}$ \\
\hline $\begin{array}{l}\text { El-Adl \& Ali } \\
\quad 2013\end{array}$ & 32 & 19.1 & $\begin{array}{l}\text { Excluded patients with } \\
\text { bone marrow disorders, } \\
\text { multiple myeloma, } \\
\text { severe anemia, patients } \\
\text { on anti-coagulation } \\
\text { therapy, radiation } \\
\text { therapy, and } \\
\text { immunosuppressive drug } \\
\text { therapy, patients with } \\
\text { diagnosed malignant } \\
\text { bone lesion, patients } \\
\text { with pathological } \\
\text { fracture, and patients } \\
\text { with less than 3-year } \\
\text { follow-up }\end{array}$ & $\begin{array}{l}\text { Benign bone } \\
\text { lesions }\end{array}$ & None & $\begin{array}{l}\text { Composite } \\
\text { ceramic graft }\end{array}$ & X Ray & $\begin{array}{l}\text { Ceraform } \\
\text { resorption and } \\
\text { bone } \\
\text { trabeculation } \\
\text { through the } \\
\text { cavitary } \\
\text { defects }\end{array}$ & 47 & $\begin{array}{l}\text { Adding bone marrow } \\
\text { aspirate to ceraform } \\
\text { biphasic ceramic had } \\
\text { hastened the rate of its } \\
\text { resorption and had } \\
\text { decreased the rate of its } \\
\text { persistence }\end{array}$ \\
\hline
\end{tabular}


Research, Society and Development, v. 10, n. 11, e94101119265, 2021

(CC BY 4.0) | ISSN 2525-3409 | DOI: http://dx.doi.org/10.33448/rsd-v10i11.19265

\begin{tabular}{|c|c|c|c|c|c|c|c|c|c|c|}
\hline $\begin{array}{c}\text { Sauerbier et } \\
\text { al. } 2011\end{array}$ & 26 & 56 & $\begin{array}{c}\text { Excluded patients } \\
\text { smoking, with history of } \\
\text { malignancy, } \\
\text { radiotherapy or } \\
\text { chemotherapy, } \\
\text { pregnancy or nursing, } \\
\text { general contraindications } \\
\text { for dental or surgical } \\
\text { treatment, medications, } \\
\text { treatments or diseases, } \\
\text { which may have an } \\
\text { effect on bone } \\
\text { remodeling, bone or } \\
\text { connective tissue } \\
\text { metabolism, or an } \\
\text { allergy to collagen }\end{array}$ & $\begin{array}{c}\text { Sinus } \\
\text { maxillary } \\
\text { augmentation }\end{array}$ & None & $\begin{array}{c}\text { Misture of } 70 \% \\
\text { of bovine bone } \\
\text { mineral and } \\
30 \% \text { of } \\
\text { autogenous } \\
\text { bone }\end{array}$ & Biopses & $\begin{array}{c}\text { New bone } \\
\text { formation } \\
\text { (Histomorpho } \\
\text { metrical } \\
\text { analysis) }\end{array}$ & 4 & Positive \\
\hline
\end{tabular}


Research, Society and Development, v. 10, n. 11, e94101119265, 2021

(CC BY 4.0) | ISSN 2525-3409 | DOI: http://dx.doi.org/10.33448/rsd-v10i11.19265

\begin{tabular}{|c|c|c|c|c|c|c|c|c|c|c|}
\hline $\begin{array}{c}\text { Pelegrine et } \\
\text { al. } 2010\end{array}$ & 13 & 47.3 & $\begin{array}{l}\text { Excluded patients with } \\
\text { systemic complications, } \\
\text { smokers, pregnant } \\
\text { woman, } \\
\text { immunocompromised } \\
\text { subjects, diabetics, and } \\
\text { the presence of sockets } \\
\text { with severe bone loss }\end{array}$ & $\begin{array}{l}\text { Alveolar } \\
\text { ridges } \\
\text { following } \\
\text { tooth } \\
\text { extraction }\end{array}$ & None & None & $\begin{array}{c}\text { Clinical } \\
\text { parameters } \\
\text { and } \\
\text { histomorpho } \\
\text { metric } \\
\text { analysis }\end{array}$ & $\begin{array}{l}\text { Alveolar } \\
\text { ridges for } \\
\text { thickness and } \\
\text { for height } \\
\text { (clinic) and } \\
\text { amounts of } \\
\text { mineralized } \\
\text { bone } \\
\text { (hismometric) }\end{array}$ & 6 & $\begin{array}{l}\text { It was observed similar } \\
\text { amounts of mineralized } \\
\text { bone but autologous } \\
\text { bone marrow graft } \\
\text { contributed to alveolar } \\
\text { bone repair after tooth } \\
\text { extraction }\end{array}$ \\
\hline
\end{tabular}

Source: Authors. 
Research, Society and Development, v. 10, n. 11, e94101119265, 2021

(CC BY 4.0) | ISSN 2525-3409 | DOI: http://dx.doi.org/10.33448/rsd-v10i11.19265

\subsection{Characteristics of the included studies related to patients}

A total of 368 patients were treated with BMA, and they had a mean age of 47.53 years. All studies included patients of both sexes. The following experimental models in medical and dental areas were evaluated: posttraumatical aseptic nonunions of long bones of the upper limb (Mazzotta et al., 2021), atrophic mandibular fracture (Manello et al., 2017), fracture of intracapsular neck femur (Verma et al., 2017), non-traumatic femoral head necrosis (Pepke et al., 206), sinus maxillary augmentation (Pasquali et al., 2015; Sauerbier et al., 2011), bilateral tibial lengthening (Lee et al., 2014), benign bone lesions (U et al., 2019; Damron et al., 2013; El-Adl et al., 2013), maxillary horizontal ridge augmentation (Da Costa et al., 2011), alveolar ridges following tooth extraction (Fontes Martins et al., 2021; Pelegrine et al., 2010). Regarding systemic conditions, one study reported that included patients submitted to chemiotherapy or immunosuppressive therapy (Pepke et al., 2016) and another one did not report systemic conditions (Mazzotta et al., 2021). Remaining studies mentioned excluded patients with pathologic fracture, polytraumatisms, or systemic conditions that would compromise the results.

\subsection{Characteristics of the included studies related to treatment and results}

Regarding the technique, most studies (seven) did not centrifugate BMA before its clinical use (U et al., 2019; Verma et al., 2017; Damron et al., 2013; El-Adl et al., 2013; Sauerbier et al., 2011; Da Costa et al., 2011; Pelegrine et al., 2010). Six studies centrifuated BMA using different protocols: Fontes Martins et al. (2021) protocol was $400 \mathrm{~g}$ for $12 \mathrm{~min}$. Mazzotta et al. (2021) was $3200 \mathrm{rpm}$ for $10 \mathrm{~min}$. Manneli et al. (2017) processed BMA at 1600g for 8 minutes. Pepke et al. (2016) followed Sepax centrifugation protocol. Pasquali et al. (2015) centrifugated it for 14 minutes. Lee et al. (2014) centrifugated BMA at $3200 \mathrm{rpm}$ for 15 minutes.

Ten studies combined BMA with another biomaterial to induce bone regeneration (Fontes Martins et al., 2021; Mazzotta et al., 2021; U et al., 2019; Manelli et al., 2017; Pasquali et al., 2015; Lee et al., 2014; Damron et al., 2013; El-Adl et al., 2013; Sauerbier et al., 2011; Da Costa et al., 2011), such as platelet-rich fibrin (Fontes Martins et al., 2021; Mazzotta et al., 2021) or platelet-rich plasma (Manelli et al., 2017; Lee et al., 2014), xenogeneic bone from bovine hydroxyapatite (Pasquali et al., 2015), ultraporous B-tricaicium phosphate (Damron et al., 2013), composite ceramic graft (El-Adl et al., 2013), misture of $70 \%$ of bovine bone mineral and 30\% of autogenous bone (Sauerbier et al., 2011) or allogeneic block grafts (Da Costa et al., 2011).

Follow-up varied from 0-47 months (mean $=15.96)$. The analyses were performed by radiography, tomography, biopsies and clinical evaluation. From 13 selected studies, nine reported a better or faster bone regeneration (primary outcomes) when BMA was used combined (Fontes Martins et al., 2021; Mazzotta et al., 2021; U et al., 2019; Manelli et al., 2017; Pasquali et al., 2015; Lee et al., 2014; El-Adl et al., 2013; Sauerbier et al., 2011; Da Costa et al., 2011) or not (Pelegrine et al., 2010) with other biomaterials. Three studies did not find advantage in the use of BMA to treat bone defects (Verma et al., 2017; Pepke et al., 2016; Damron et al., 2013). All selected studies also evaluated other parameters (secondary results) related to healing, such as postoperative complications and pain visual analogue score. There were no adverse or negative effects of BMA reported.

\section{Discussion}

One of the methods able to improve healing of damaged tissue is the use of cellular based therapies. Expanded cultured mesenchymal stem cells (MSCs), bone marrow concentrate and bone marrow in toto, or bone marrow aspirate (BMA), represent the main strategies developed and used for tissue regeneration (Lana et al., 2021; Salamanna et al., 2018). 
Research, Society and Development, v. 10, n. 11, e94101119265, 2021

(CC BY 4.0) | ISSN 2525-3409 | DOI: http://dx.doi.org/10.33448/rsd-v10i11.19265

Studies have demonstrated that MSC derived from bone marrow have a higher osteogenic potential than those from other anatomical origins (Schneider et al., 2010; Zhang et al., 2009). Also, it is clear in the literature that its in vitro expansion phase causes dramatic changes in MSC phenotype, what has very significant implications for the development of effective therapies (Bara et al., 2014). So, genuine use of BMA is a very interesting way to apply MSC in surgical sites to promote bone healing. In recent years, there has been much research on this topic, but the benefits of BMA are still controversial. Thus, we systematically investigated the results published on the effect of this therapeutic approach on bone formarion in the health field.

The present review included only studies that clinically evaluated bone defects treated with BMA and compared to a control group. Most studies evaluated the results of bone formation radiographically (primary outcomes). In the qualitative analysis, most of the included studies presented positive primary or secondary outcomes with BMA treatment in bone defects (Martins et al., 2021; Mazzotta et al., 2021; U et al., 2019; Manelli et al., 2017; Pasquali et al., 2015; Lee et al., 2014; Damron et al., 2013; El-Adl et al., 2013; Sauerbier et al., 2011; Da Costa et al., 2011; Pelegrine et al., 2010). These studies demonstrated that BMA can be a useful tool to promote bone formation. It is possible that the positive bone regeneration results observed in groups treated with BMA in the selected studies were due to a significant increase in MSC at the surgical site (Santinoni et al., 2021; Nagata et al., 2013). This hypothesis is supported by the findings of Smiler et al. (2008) who analyzed samples of BMA of patients aged 23-73 years by flow cytometry analysis. The authors used cell markers for stem cells and observed that BMA contains a significantly amount of hematopoietic, endothelial and MSC.

However, some authors believe that BMA alone is not enough use unmodified as a source of MSC (Schneider et al., 2010; Kraus t al., 2006). Age related changes in the bone marrow microenvironment result in a decrease in the number and function of MSC that differentiate into bone forming osteoblasts (Duque, 2008). The MSC pool can also be depleted in younger individuals because of radiotherapy for cancer (Greenberger \& Epperly, 2009). Thus, a reduction in endogenous MSC due to advancing age or an underlying medical condition will also compromise bone regeneration (Gao et al., 2012; Chines, 2010). In fact, three studies included in this review reported no improvement with the treatment (Verma et al., 2017; Pepke et al., 2016; Damron et al., 2013). Verma et al. (2017) used BMA to treat fracture of intracapsular neck femur. The authors did not perform any processing of BMA and did not associate another biomaterial to it. Evaluated groups were closed reduction and cannulated cancellous screw fixation (Group A) and additional percutaneous autologous bone marrow aspirate instillation at fracture site was done along with cannulated cancellous screw fixation (Group B). Results were evaluated until 19.6 months (mean follow up) and mean age of the patients was 39.5 years. They have not found significant difference between test and control groups. Pepke et al. (2016) evaluated healing of non-traumatic femoral head necrosis with injection of BMA concentrate (BMAC) during core decompression in comparison to core decompression only. BMA processing followed Sepax centrifugation device protocol. Results were evaluated until 24 months follow up and mean age of the patients was 44.4 years. It was not observed any benefit from the injection of bone marrow concentrate with regard to bone regeneration and clinical outcomes. Damron et al. (2013) treated benign bone lesion healing filled with ultraporous $\beta$-tricaicium phosphate (TCP) versus TCP plus BMA (TCP/BMA) with the hypothesis that BMA speeds incorporation of bone graft substitute. The authors did not perform any processing of BMA and found that the addition of BMA did not provide any significant benefit. Findings were analyzed until 19 months (mean follow up) and mean age of the patients was 23.5 years.

These results demonstrate that centrigugation, combination with other biomaterials, or patient age could not absolutely influence the results of BMA on bone healing. The main subjects that seem to influence the results was the systemic condition of the patients and experimental models involved. While the study by Pepke et al. (2016) involved patients submitted to chemotherapy or immunosuppressive therapy and did not find positive results in groups treated with BMA, other studies had 
found positive results and excluded patients that had a history of neoplastic disease treated with radiotherapy or chemotherapy, if they were pregnant or breastfeeding, if they were receiving treatment or were affected by an illness that could have a change on bone homeostasis, and other that coul affect healing (U et al., 2019; Manelli et al., 2017; Pasquali et al., 2015; Lee et al., 2014; Damron et al., 2013; El-Adl et al., 2013; Sauerbier et al., 2011; Da Costa et al., 2011; Pelegrine et al., 2010).

Finally, is important to highlight the secondary outcomes, which also had positive results after BMA treatment in bone defects. Besides the amount of bone formed have been greater in the test groups with BMA, other advantages related to the acceleration of healing, decreasing the postoperative morbidity of the patients, and the shorter time for reabsorption of biomaterials were also observed as secondary results of this treatment. This therapy can show a benefit to the population, even for elderly patients or for whom additional care is required (Santinoni et al., 2017).

\section{Conclusion}

Within the limitation of this review, an improvement in the healing can be found when BMA is used to treat bone defects if patients had not a history of radiotherapy, chemotherapy or suppressive treatment. BMA should be be further evaluated in comparison with autologous bone graft.

\section{References}

Badiavas, E. V., \& Falanga. V. (2013). Treatment of chronic wounds with bone marrow-derived cells. Arch. Dermatol. 139: 510-516.

Bansal, S., Chauhan, V., Sharma, S., Maheshwari, R., Juyal, A., \& Raghuvanshi, S. (2009). Evaluation of hydroxyapatite and beta-tricalcium phosphate mixed with bone marrow aspirate as a bone graft substitute for posterolateral spinal fusion. Indian J. Orthop. 43: 234-239.

Bara, J. J., Richards, R. G., Alini, M., \& Stoddart, M. J. (2014). Concise review: bone marrow-derived mesenchymal stem cells change phenotype following in vitro culture: implications for basic research and the clinic. Stem Cells. 32: 1713-1723.

Burastero, G., Scarfi, S., Ferraris, C., Fresia, C., Sessarego, N., Fruscione, F., Monetti, F., Scarfò, F., Schupbach, P., Podestà, M., Grappiolo, G., \& Zocchi, E. (2010). The association of human mesenchymal stem cells with BMP-7 improves bone regeneration of critical-size segmental bone defects in athymic rats. Bone. 47: 117-126.

Clines, G. A. (2010). Prospects for Osteoprogenitor Stem Cells in Fracture Repair and Osteoporosis. Curr. Opin. Organ. Transpl. 15: 73-78.

Clough, B. H., McCarley, M. R., Krause, U., Zeitouni, S., Froese, J. J., McNeill, E. P., Chaput, C. D., Sampson, H. W., \& Gregory, C. A. (2015). Bone regeneration with osteogenically enhanced mesenchymal stem cells and their extracellular matrix proteins. J. Bone Miner. Res. 30: 83-94.

Da Costa, C. E. S., Pelegrine, A. A., Fagundes, D. J., Simoes, M. de J., \& Taha, M. O. (2011). Use of corticocancellous allogeneic bone blocks impregnated with bone marrow aspirate: A clinical, tomographic, and histomorphometric study. Gen. Dent. 59: e200-e205.

Damron, T. A., Lisle, J., Craig, T., Wade, M., Silbert, W., \& Cohen, H. (2013). Ultraporous B-tricalcium phosphate alone or combined with bone marrow aspirate for benign cavitary lesions. J Bone Joint Surg Am. 95: 158-166.

Duque, G. (2008). Bone and fat connection in aging bone. Curr. Opin. Rheumatol. 20: 429-434.

El-Adl, G., \& Ali, A. M. (2013). Does bone marrow affect the radiological outcome when added to biphasic ceramic graft in treatment of benign bone lesions?. Eur. J. Orthop. Surg. Traumatol. 23: 13-20.

Fontes Martins, L. C., Sousa Campos de Oliveira, A. L., Aloise, A. C., Scavone de Macedo, L. G., Teixeira, M. L., Moy, P. K., \& Pelegrine, A. A. (2021). Bone marrow aspirate concentrate and platelet-rich fibrin in fresh extraction sockets: A histomorphometric and immunohistochemical study in humans. $J$. Craniomaxillofac. Surg. 49: 104-109.

Gao, C., Gao, Q., Li, Y., Rahaman, M.N., Teramoto, A., \& Abe, K. (2012). Preparation and in vitro characterization of electrospun PVA scaffolds coated with bioactive glass for bone regeneration. J. Biomed. Mater. Res. Part A. 100: 1324-1334.

Greenberger, J. S., \& Epperly, M. (2009). Bone Marrow-Derived Stem Cells and Radiation Response. Semin. Radiat. Oncol. 19: 133-139.

Gupta, G. J., Karki, K., Jain, P., \& Saxena, A. K. (2017). Autologous Bone Marrow Aspirate Therapy for Skin Tissue Engineering and Tissue Regeneration. Adv. Wound Care. 6: 135-142.

Harada, N., Watanabe, Y., Sato, K., Abe, S., Yamanaka, K., Sakai, Y., Kaneko, T., \& Matsushita, T. (2014). Bone regeneration in a massive rat femur defect through endochondral ossification achieved with chondrogenically differentiated MSCs in a degradable scaffold. Biomaterials. 35: 7800-7810. 
Research, Society and Development, v. 10, n. 11, e94101119265, 2021 (CC BY 4.0) | ISSN 2525-3409 | DOI: http://dx.doi.org/10.33448/rsd-v10i11.19265

He, N., Zhang, L., Cui, J., \& Li, Z. (2014). Bone Marrow Vascular Niche: Home for Hematopoietic Stem Cells. Bone Marrow Res. $2014: 128436$.

Isern, J., \& Méndez-Ferrer, S. (2011). Stem cell interactions in a bone marrow niche. Current. Osteoporos. Rep. 9: $210-218$.

Jadad, A. R., Moore, R. A., Carroll, D., Jenkinson, C., Reynolds, D. J., Gavaghan, D. J., \& McQuay, H. J. (1996). Assessing the quality of reports of randomized clinical trials: Is blinding necessary? Control. Clin. Trials. 17: 1-12.

Jäger, M., Herten, M., Fochtmann, U., Fischer, J., Hernigou, P., Zilkens, C., Hendrich, C., \& Krauspe, R. (2011). Bridging the gap: Bone marrow aspiration concentrate reduces autologous bone grafting in osseous defects. J. Orthop. Res. 29: 173-180.

Kaigler, D., Pagni, G., Park, C.H., Tarle, S.A., Bartel, R.L., Giannobile, W.V. (2010). Angiogenic and Osteogenic Potential of Bone Repair Cells for Craniofacial Regeneration. Tissue Eng. Part A. 16: 2809- 2820.

Kraus, K. H., \& Kirker-Head, C. (2006). Mesenchymal stem cells and bone regeneration. Vet. Surg. 35: 232-242.

Lana, J. F., da Fonseca, L. F., Azzini, G., Santos, G., Braga, M., Cardoso Junior, A. M., Murrell, W. D., Gobbi, A., Purita, J., \& Percope de Andrade, M. A. (2021). Bone Marrow Aspirate Matrix: A Convenient Ally in Regenerative Medicine. Int. J. Mol. Sci. 22: 2762.

Landis, J. R., \& Koch, G. G. (1977). The Measurement of Observer Agreement for Categorical Data. Biometrics. 33: 159-174.

Lee, D. H., Ryu, K. J., Kim, J. W., Kang, K. C., \& Choi, Y. R. (2014). Bone Marrow Aspirate Concentrate and Platelet-rich Plasma Enhanced Bone Healing in Distraction Osteogenesis of the Tibia. Clin. Orthop. Rel. Res. 472: 3789-3797.

Lemos, C. A. A., Mello, C. C., dos Santos, D. M., Verri, F. R., Goiato, M. C., \& Pellizzer, E. P. (2016). Effects of platelet-rich plasma in association with bone grafts in maxillary sinus augmentation: a systematic review and meta-analysis. Int. J. Oral Maxillofac. Surg. 45: 517-525.

Mannelli, G., Arcuri, F., Conti, M., Agostini, T., Raffaini, M., \& Spinelli, G. (2017). The role of bone marrow aspirate cells in the management of atrophic mandibular fractures by mini-invasive surgical approach: Single-institution experience. J. Cranio-Maxillofacial Surg. 45: 694-703.

Mazzotta, A., Stagni, C., Rocchi, M., Rani, N., Del Piccolo, N., Filardo, G., \& Dallari, D. (2021). Bone marrow aspirate concentrate/platelet-rich fibrin augmentation accelerates healing of aseptic upper limb nonunions. J. Orthop. Traumatol. 22: 21.

Moher, D., Liberati, A., Tetzlaff, J., Altman, D. G., \& PRISMA Group. (2010). Preferred reporting items for systematic reviews and meta-analyses (PRISMA) statement and publication bias. Int. J. Surg. 8: 336-341.

Nagata, M. J., Santinoni, C. S., Pola, N. M., de Campos, N., Messora, M. R., Bomfim, S. R., Ervolino, E., Fucini, S. E., Faleiros, P. L., Garcia, V. G., Bosco, A. F. (2013). Bone marrow aspirate combined with low-level laser therapy: A new therapeutic approach to enhance bone healing. J. Photochem. Photobiol. B Biol. 121: 6-14.

Oliveira, H. F. E., Verri, F., Lemos, C. A., Cruz, R., Batista, V. E. S., Pellizzer, E., Santinoni, C. (2020). Clinical Evidence for Treatment of Class II Periodontal Furcation Defects. Systematic Review and Meta-analysis. J. Int. Acad. Periodontol. 22:117-128.

Pasquali, P. J., Teixeira, M. L., de Oliveira, T. A., de Macedo, L. G., Aloise, A. C., Pelegrine, A. A. (2015). Maxillary Sinus Augmentation Combining BioOss with the Bone Marrow Aspirate Concentrate: A Histomorphometric Study in Humans. Int. J. Biomater. 2015 : 121286.

Pelegrine, A. A., da Costa, C. E., Correa, M. E., Marquesm J. F. Jr. (2010). Clinical and histomorphometric evaluation of extraction sockets treated with an autologous bone marrow graft. Clin. Oral Implants. Res. 21: 535-542.

Pelegrine, A. A., Teixeira, M. L., Sperandio, M., Almada, T. S., Kahnberg, K. E., Pasquali, P. J., Aloise, A. C. (2016). Can bone marrow aspirate concentrate change the mineralization pattern of the anterior maxilla treated with xenografts? A preliminary study. Contemp. Clin. Dent. 7: 21-26.

Pepke, W., Kasten, P., Beckmann, N.A., Janicki, P., Egermann, M. (2016). Core decompression and autologous bone marrow concentrate for treatment of femoral head osteonecrosis: A randomized prospective study. Orthop Rev. (Pavia). 8: 6162.

Pittenger, M. F., Mackay, A. M., Beck, S. C., Jaiswal, R. K., Douglas, R., Mosca, J. D., Moorman, M. A., Simonetti, D. W., Craig, S., Marshak, D. R. (1999). Multilineage Potential of Adult Human Mesenchymal Stem Cells. Science. 284: 143-147.

Salamanna, F., Contartese, D., Nicoli Aldini, N., Barbanti Brodano, G., Griffoni, C., Gasbarrini, A., Fini, M. (2018). Bone marrow aspirate clot: A technical complication or a smart approach for musculoskeletal tissue regeneration? J. Cell Physiol. 233: 2723-2732.

Santinoni, C. D., Oliveira, H. F., Batista, V. E., Lemos, C. A., Verri, F. R. (2017). Influence of low-level laser therapy on the healing of human bone maxillofacial defects: A systematic review. J. Photochem. Photobiol. B Biol. 169: 83-89.

Santinoni, C. S., Neves, A. P. C., Almeida, B. F. M., Kajimoto, N. C., Pola, N. M., Caliente, E. A., Belem, E. L. G., Lelis, J. B., Fucini, S. E., Messora, M. R., Garcia, V. G., Bomfim, S. R. M., Ervolino, E., Nagata, M. J. H. (2021). Bone marrow coagulated and low-level laser therapy accelerate bone healing by enhancing angiogenesis, cell proliferation, osteoblast differentiation, and mineralization. J. Biomed. Mater. Res. A. 109: 849-858.

Sauerbier, S., Rickert, D., Gutwald, R., Nagursky, H., Oshima, T., Xavier, S. P., Christmann, J., Kurz, P., Menne, D., Vissink, A., Raghoebar, G., Schmelzeisen, R., Wagner, W., Koch, F. P. (2011). Bone Marrow Concentrate and Bovine Bone Mineral for Sinus Floor Augmentation: A Controlled, Randomized, Single-Blinded Clinical and Histological Trial-Per-Protocol Analysis. Tissue Eng. Part A. 17: $2187-2197$.

Schneider, R. K., Puellen, A., Kramann, R., Raupach, K., Bornemann, J., Knuechel, R., Pérez-Bouza, A., Neuss, S. (2010). The osteogenic differentiation of adult bone marrow and perinatal umbilical mesenchymal stem cells and matrix remodelling in three-dimensional collagen scaffolds. Biomaterials. 31: 467480 . 
Research, Society and Development, v. 10, n. 11, e94101119265, 2021

(CC BY 4.0) | ISSN 2525-3409 | DOI: http://dx.doi.org/10.33448/rsd-v10i11.19265

Siegel, H. J., Baird, R. C. 3rd, Hall, J., Lopez-Ben, R., Lander, P. H. (2008). The outcome of composite bone graft substitute used to treat cavitary bone defects. Orthopedics. 31: 754 .

Smiler, D., Soltan, M., Albitar, M. (2008). Toward the Identification of Mesenchymal Stem Cells in Bone Marrow and Peripheral Blood for Bone Regeneration. Implant Dent. 17: 236-274.

Soltan, M., Smiler, D., Prasad, H. S., Rohrer, M. D. (2017). Bone Block Allograft Impregnated with Bone Marrow Aspirate. Implant Dent. 16: $329-339$.

Tewari, D., Khan, M. P., Sagar, N., China, S. P., Singh, A. K., Kheruka, S. C., Barai, S., Tewari, M. C., Nagar, G. K., Vishwakarma, A. L., Ogechukwu, O. E., Bellare, J. R., Gambhir, S., Chattopadhyay, N. (2015). Ovariectomized Rats with Established Osteopenia have Diminished Mesenchymal Stem Cells in the Bone Marrow and Impaired Homing, Osteoinduction and Bone Regeneration at the Fracture Site. Stem Cell Rev. Reports. 11: 309-321.

U, V., Mehrotra, D., Howlader, D., Kumar, S., Anand, V. (2019). Bone Marrow Aspirate in Cystic Maxillofacial Bony Defects. J. Craniofac. Surg. 30: e247e251.

Verma, N., Singh, M. P., Ul-Haq, R., Rajnish, R. K., Anshuman, R. (2017). Outcome of bone marrow instillation at fracture site in intracapsular fracture of femoral neck treated by head preserving surgery. Chinese J. Traumatol. 20: 222-225.

Weel, H., Mallee, W. H., Van Dijk, C. N., Blankevoort, L., Goedegebuure, S., Goslings, J. C., Kennedy, J. G., Kerkhoffs, G. M. (2015). The effect of concentrated bone marrow aspirate in operative treatment of fifth metatarsal stress fractures, a double-blind randomized controlled trial. BMC Musculoskelet. Disord. 16: 211.

Zhang, Z. Y., Teoh, S. H., Chong, M. S., Schantz, J. T., Fisk, N. M., Choolani, M. A., \& Chan, J. (2009). Superior Osteogenic Capacity for Bone Tissue Engineering of Fetal Compared with Perinatal and Adult Mesenchymal Stem Cells. Stem Cells. 27: 126-137. 